KARL JANSEN-WINKELN

\title{
Eine Familie im Totenkult
}

\author{
Hierzu Tafel XXVII
}

Seit langer Zeit schon ist im Ägyptischen Museum Kairo das Fragment einer Stele ${ }^{1}$ ausgestellt, das A. Mariette in der nördlichen Nekropole von Abydos gefunden hatte ${ }^{2}$. Es handelt sich um ein max. $70 \mathrm{~cm}$ breites und $52 \mathrm{~cm}$ hohes Stück vom oberen Teil einer ursprünglich recht großen Stele, die einem Priester namens $\underline{D} d-J n j-$ hrt-jw.f-enh gehört (Abb. 1 und Taf. XXVII). Vom Haupttext sind nur Reste der ersten sechs Zeilen erhalten, vermutlich beginnend mit einer htp-dj-njswt-Formel ${ }^{3}$. Über dem Haupttext befindet sich die Darstellung einer Art Prozession von (ursprünglich) zwölf Personen, nach rechts gewandt, von der die acht hinteren eine Götterstatue tragen, während die vier vorderen Götterstandarten und andere Kultgegenstände mit sich führen. Über dem Blickfeld sind 29 Kolumnen Text, in vier Blöcke gegliedert, mit insgesamt 13 Beischriften zu dieser Darstellung, jeweils von $d d-m d w$ eingeleitet, und zwar zu den zwölf Personen sowie zu der Götterstatue.

Dieses Fragment ist 1880 von A. Mariette in zeichnerischer Wiedergabe veröffentlicht wor$\operatorname{den}^{4}$ und in dem im gleichen Jahr erschienenen Katalog der Funde beschrieben und besprochen

Temporary Number 30/5/24/1. Ich danke dem ehemaligen Generaldirektor des Ägyptischen Museums Kairo, Herrn Prof. Dr. M. Saleh, herzlich für seine Genehmigung zur Wiedergabe des Fotos (Aufnahme Museum Kairo) und für die Möglichkeit, den Text zu kollationieren.

${ }^{2}$ Vgl. PM V, 59.

${ }^{3}$ Was aber natürlich nicht heißt, daß der ganze Text aus einer solchen Formel bestand. Totenopferformeln können ja als Einleitung ganz unterschiedlicher Textsorten dienen, wie z. B. Hymnen, Verklärungen, Gebete, Anrufe an die Lebenden, Biographien etc. pl. 58 .

Abydos, Description des fouilles, II, Paris 1880 ,

worden $^{5}$. Allerdings ist die Zeichnung merkwürdigerweise seitenverkehrt, und die Wiedergabe der Inschriften enthält eine ganze Reihe von Fehlern und Auslassungen. Sein Übersetzungsversuch für die Rede des $N s-M n w$ ist dementsprechend auch mißglückt ${ }^{6}$.

In neuerer Zeit ist die Stele zweimal ausführlicher behandelt worden: 1954 hat H. de Meulenaere darauf hingewiesen, daß demselben $\underline{D} d$ Jnj-hrt-jw.f- $n h$ auch eine Stele in der ägyptischen Sammlung in Leiden (VII,9) gehört? Beide Denkmäler zusammen ergeben einen ausführlichen, über sechs Generationen reichenden Stammbaum dieser Familie. Diese prosopographischen Daten und v. a. die Titel der Personen sind von de Meulenaere ausführlich erörtert worden. 1973 hat P. Munro das Fragment in Kairo im Rahmen seiner Gesamtdarstellung der Totenstelen der Spätzeit besprochen ${ }^{8}$, allerdings ohne Abbildung. Davon abgesehen ist die Stele weitgehend unbeachtet geblieben ${ }^{10}$.

Wie so viele Denkmäler der Spätzeit, sind auch die Stelen des $\underline{D} d-J n j-h r t-j w . f-\ulcorner h h$ schwierig zu datieren. De Meulenaere hält - sehr vorsichtig - den Zeitraum vom Ende des 26. Dynastie

Catalogue général des monuments d'Abydos, Paris 1880, 489-92 (No. 1297).

Catalogue général, 490-1. 221-36

„Une famille des prêtres thinites“, CdE 29, 1954,

P. Munro, Die spätägyptischen Totenstelen, ÄF 25, 1973, 111-2; 303-4 (und die Stele Leiden VII, 9 auf p. 300; Taf. 44).

Ein kleines Foto der Stele, das einzige bis jetzt erschienene, bei F. W. von Bissing, Die Kultur des alten Ägyptens, Leipzig 1913, Taf. 21, Abb. 54.

Eine Erwähnung bei M. J. Raven, OMRO 60, 1979, 284 (mit n. 237). 


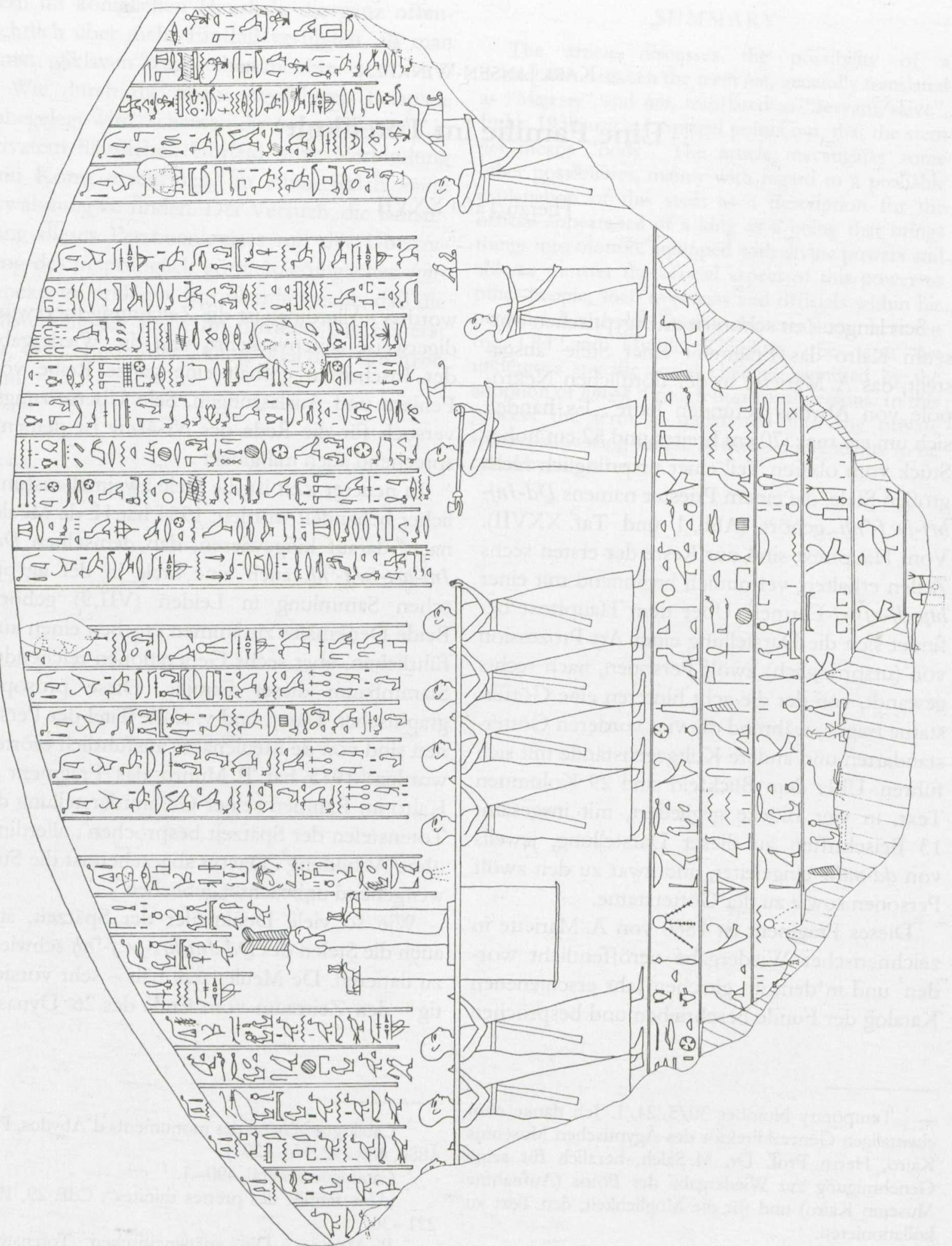

Abb. 1. Stele des Dd-Jnj-hrt-jw f- $n h$ (II), Kairo TN 30/5/24/1 


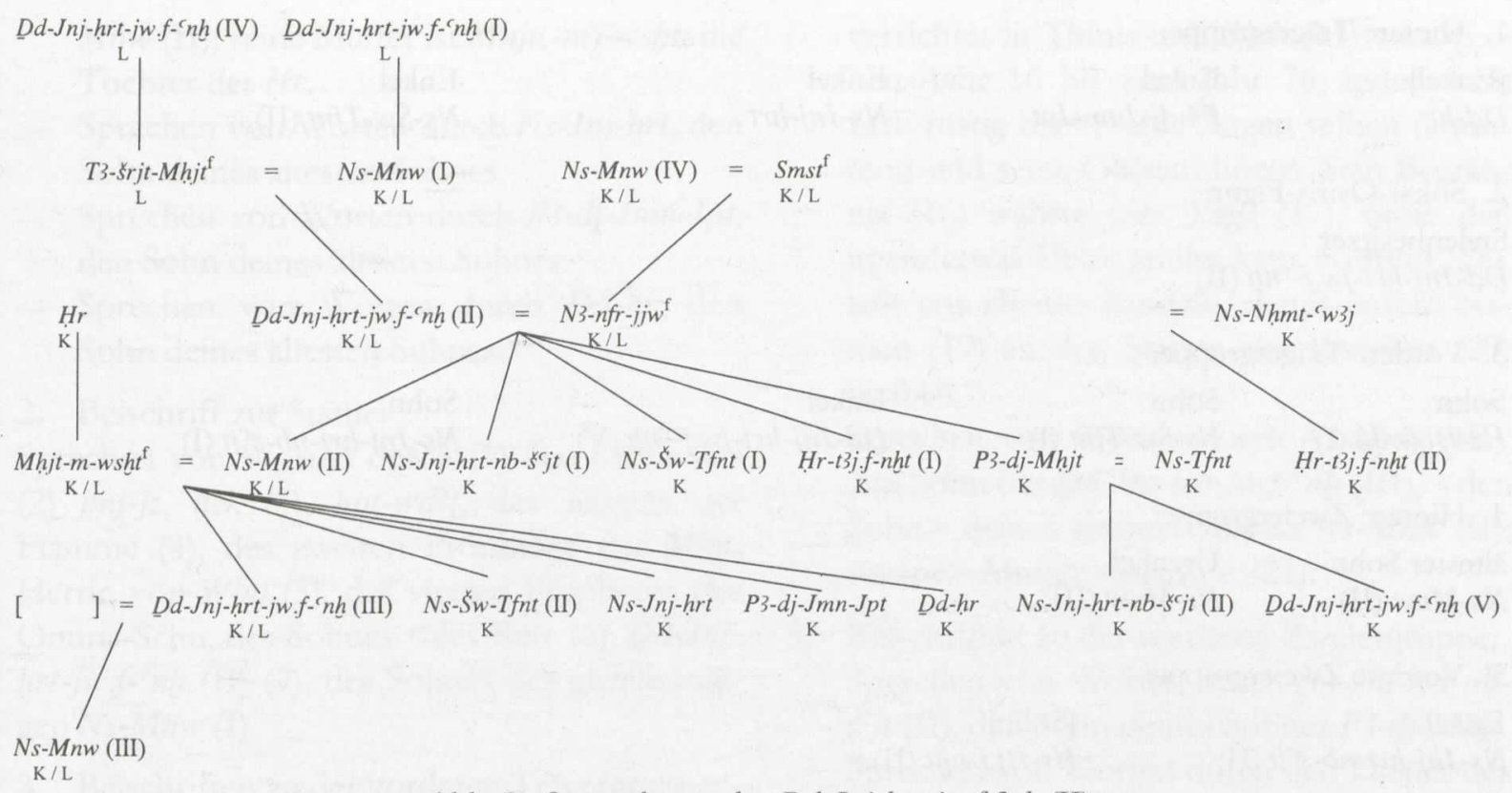

Abb. 2. Stammbaum des $\underline{D} d-J n j-h r t-j w . f-n h h$ (II)

bis zu den ersten Ptolemäern für möglich ${ }^{11}$, Munro spricht sich für eine Datierung um 300 v. Chr. aus. Ein solch genauer Ansatz ist aber vielleicht doch etwas zu optimistisch, das gesamte 4. Jahrhundert und der Beginn des 3. dürfte - als eine besonders experimentierfreudige Epoche mit zahlreichen Denkmälern - vorläufig in Betracht kommen.

Den Stammbaum der Familie, so wie er sich aus beiden Stelen ergibt, hat de Meulenaere schon dargestellt ${ }^{12}$. Allerdings ist diese Genealogie $\mathrm{m}$. E. in einem Punkt zu berichtigen. Auf dem Fragment in Kairo kommt zweimal ein Sohn des Stelenbesitzers namens $H r$ - $t 3 j . f-n h t$ vor: der unmittelbar vor der Götterfigur gehende Träger nennt sich z3.k Hr-t3j.f-nht, mwt.f N(3)$n f r-j j w$, und der die ganze Prozession anführende Priester (von dem nur noch die Beischrift erhalten ist) wird als $z 3 . k H r-t 3 j . f-n h t, m w t . f N s-$ $N h m t-{ }^{-} w 3 j$ bezeichnet. De Meulenaere hielt es offenbar für unwahrscheinlich, daß ein Mann zwei gleichnamige Söhne hat, denn er identifiziert die beiden miteinander und muß dementsprechend $N s-N h m t-{ }^{\ulcorner} w 3 j$ für einen Beinamen der N3-nfr-jjw halten ${ }^{13}$. Da aber beide Personen

CdE 29, 236.

${ }^{12} \mathrm{CdE} 29,225$.

${ }^{13}$ Bzw. ein Versehen im Text annehmen, vgl. CdE $29,223$. nicht nur Mütter mit verschiedenen Namen haben, sondern auch an unterschiedlicher Stelle der Prozession dargestellt werden, halte ich es für ganz sicher, daß es sich um zwei distinkte Personen handelt (die man in der Praxis ja leicht als „der Große“ und „der Kleine“ hätte unterscheiden können).

Es ergibt sich also folgender Stammbaum (Abb. 2) $)^{14}$. Von diesen Familienmitgliedern sind im Bildfeld der Stele aus Kairo alle Söhne des Stelenbesitzers mit Ausnahme des P3-dj-Mhjt dargestellt, der vermutlich schon tot ist, ebenso die Söhne des ältesten Sohnes Ns-Mnw II mit Ausnahme des $\underline{D} d$-Jnj-hrt-jw.f- $n h$ II, der ebenfalls schon tot sein könnte, denn er wird von seinem Sohn Ns-Mnw III (also dem Urenkel des Stelenbesitzers) vertreten. Entsprechend der Gliederung der Beischriften sind auch bei der Darstellung neben der Götterfigur vier Gruppen zu unterscheiden, die jeweils von bestimmten Familienmitgliedern gebildet werden ${ }^{15}$ :

${ }^{14}$ Die Numerierung nach de Meulenaere; die Zusätze $\mathrm{K}$ und $\mathrm{L}$ je nachdem, ob die betreffende Person auf den Stelen in Kairo und/oder in Leiden bezeugt sind.

${ }^{15} \mathrm{Im}$ folgenden von links nach rechts, d. h. von hinten nach vorn aufgezählt, entsprechend der Darstellung. 
1. Hintere Trägergruppe:

$\begin{array}{llll}\text { [Enkel] } & \text { Enkel } & \text { Enkel } & \text { Enkel } \\ \underline{D} d-h r & P 3-d j-J m n-J p t & N s-J n j-h r t & N s-S ̌ w-T f n t \text { (II) }\end{array}$

2. Sokar-Osiris-Figur:

Stelenbesitzer

$\underline{D} d-J n j-h r t-j w . f-r n h \underline{\text { (II) }}$

3. Vordere Trägergruppe:

Sohn Sohn

$H r-t 3 j: f-n h t(\mathrm{I}) \quad$ Ns-Šw-Tfnt (I)

Enkel

Sohn

4. Hintere Zweiergruppe:

ältester Sohn Urenkel

Ns-Mnw (II) Ns-Mnw (III)

5. Vordere Zweiergruppe:

Enkel

Ns-Jnj-hrt-nb-šjt (II)

[Sohn]

Hr-t3j.f-nht (II)

Die hinter der Götterfigur gehenden Träger sind alle Söhne des ältesten Sohnes Ns-Mnw II, die Gruppe der vier Träger davor besteht aus drei Söhnen des Toten von seiner Frau N3-nfr$j j w$ und einem Enkel, der offenbar seinen schon verstorbenen Vater $P 3-d j-M h j t$ vertritt. Man wird daraus schließen dürfen, daß auch $P 3$-dj-Mhjt ein Sohn der N3-nfr-jjw war. Die Gruppe der vorderen Träger besteht also aus den direkten Nachkommen des Stelenbesitzers mit Ausnahme seines ältesten Sohnes (und mutmaßlichen Haupterben). Dieser älteste Sohn geht unmittelbar vor der Trägergruppe, eine Götterstandarte und einen Räucherarm tragend, und vor ihm befindet sich sein Enkel, der Sohn seines (schon verstorbenen ältesten?) Sohnes $\underline{D} d-J n j-h r t-j w . f-{ }^{\prime} n h$ (III). Diese Gruppe umfaßt dann wohl die Haupterben des Toten. Die beiden Männer an der Spitze des Zuges scheinen dagegen gewissermaßen die „Außenseiter" der Familie zu sein: der einzige von einer zweiten Frau stammende Sohn (Hr-t3j.f-nht II) und ein weiterer Enkel, der nicht der Sohn des ältesten Sohnes des Toten ist. Zumindest $H r$-t3j.f-nht (II) hat aber für das Begräbnis seines Vaters eine wichtige Funktion gehabt, wie die Beischrift verrät (s. u.).

Es gibt insgesamt 13 Beischriften, zu jeder der dargestellten Personen und zusätzlich zu der Götterfigur, die durch den Text über ihr als der tote Stelenbesitzer identifiziert wird. Diese den Toten repräsentierende Figur ist das Zentrum der Szene. Zum einen sind die Hieroglyphen aller Beischriften auf diese Statue hin orientiert (die Beischrift der Statue selbst hat die gleiche Blickrichtung wie sie), zum anderen sind alle Beischriften in der Anredeform gehalten ( $\underline{d} d-m d w$ jn $z 3 . k$ ...u. a..), was eigentlich recht unüblich ist. Der Stelenbesitzer wird in der Beischrift zur Götterfigur mit seinen Titeln bezeichnet, während die Teilnehmer der Prozession allein durch ihre Namen und ihre verwandtschaftliche Beziehung zum Toten identifiziert werden (nur der Sohn $H r$-t3j.f-nht II führt einen - sehr ungewöhnlichen - Titel, der vielleicht mit seiner besonderen Funktion zusammenhängt). Obwohl alle Beischriften mit $\underline{d} d-m d w$ jn beginnen ${ }^{16}$, werden nur dem ältesten Sohn Ns-Mnw II und dem Sohn $H r-t 3 j . f-n h t$ II eigene Reden in den Mund gelegt.

\section{Übersetzung}

\section{A. Stelenrund}

1. Beischrift zu der hinteren Trägergruppe:

- Sprechen von Worten durch (1) Ns-Šw-Tfnt (II), den Sohn deines ältesten Sohnes Ns-

${ }^{16}$ Bei den letzten drei Personen links ist die Gruppe $d d-m d w$ jn auf den oberen Rand der Stele geschrieben und daher vielleicht nachträglich hinzugefügt worden. 
Mnw (II); seine Mutter ist Mhjt(-m)-wsht, die Tochter des $H r$.

- Sprechen von Worten durch Ns-Jnj-hrrt, den Sohn deines ältesten Sohnes.

- Sprechen von Worten durch P3-dj-Jmn-Jpt, den Sohn deines ältesten Sohnes.

- Sprechen von Worten durch $\underline{D} d-h r$, den Sohn deines ältesten Sohnes.

2. Beischrift zur Statue:

Sprechen von Worten durch (1) den Osiris des (2) $j m j-j z, h z k$ (3), $h p t-w d \underline{d} t$, des Trägers der Flamme (4), des zweiten Propheten der Mhjt, Herrin von Wbn (5), des vierten Propheten des Onuris-Schu, des Sohnes <des Re> (6), D $d$-Jnjhrt-jw.f- $n h$ (II) (7), des Sohnes des gleichrangigen Ns-Mnw (I)

3. Beischriften zu der vorderen Trägergruppe:

- Sprechen von Worten durch deinen Sohn $H r$-t3j.f-nht (I); seine Mutter ist N(3)-nfr-jjw.

- Sprechen von Worten durch deinen Sohn $N s-\breve{S} w-T f n t(I)$; seine Mutter ist N3-nfr-jjw.

- Sprechen von Worten durch Dd-Jnj-hrtjw. f- $n h(\mathrm{~V})$, den Sohn deines Sohnes P3-dj$M h j t ;<$ seine $>$ Mutter ist $N s-T f<n t>$.

- Sprechen von Worten durch deinen Sohn Ns-Jnj-hrt-nb-šjt (I); seine Mutter ist N3$n f r-j j w$, die Tochter des Propheten der Mhjt, der Herrin von Wbn, Ns-Mnw (IV); ihre Mutter ist Smst.

4. Beischriften zu der hinteren Zweiergruppe:

- Sprechen von Worten durch deinen ältesten Sohn Ns-Mnw (II) zu seinem ganzen ,Kollegium' ebenso wie (zu) seinen Kindern (8): „Legt eure Arme unter euren Vater (9) in T3-wr! Eure Arme sollen nicht erlahmen, wenn ihr ihn tragt (10), um ihn nach Thinis und Bhdt (11) gelangen zu lassen im Verlauf jeden Tages. Vollzieht ihm seine Riten vor den Göttern in ihnen (12), so wie er (es) für sie (13) getan hat auf Erden. Denn ein Ehrwürdiger bei seinem Gott, ein Privilegierter bei seinen Mitbürgern (14), er hat Zutritt zu Gott, er heiligt das Geheimnis seines Gottes, er legt die Ehrfurcht vor ihm jedermann auf. (Ich) will euch über das Geschehene berichten, damit ihr (eurerseits) eifrig seid wegen dessen, was er für die Götter seines Gaus getan hat: Er hat seinen Dienst (15) verrichtet in Thinis und $B h d t$ (11) vom (Lebens)Jahr 16 bis zum Jahr 76, indem sein Leib rüstig blieb, seine Augen se[hen (konnten)] und seine $\mathrm{Oh}[\mathrm{ren}]$ hören. Sein Begräbnis (16) währte vier Tage (17), ohne daß irgendetwas Übles an ihn kam. Kommt (18), laßt uns ebenso handeln, damit unsere $\mathrm{Na}$ men (19) an den Stätten der Ewigkeit (20) bleiben."

- Sprechen von Worten durch Ns-Mnw (III), den Sohn des $\underline{D} d$-Jnj-hrt-jw.f- $n h$ (III), <den Sohn> deines ältesten Sohnes Ns-Mnw (II); $<$ seine $>$ Mutter $<$ ist XY> (21).

5. Beischriften zu der vorderen Zweiergruppe: - Sprechen von Worten durch Ns-Jnj-hrt-nbšjt (II), den Sohn deines Sohnes P3-dj-Mhjt.

- Sprechen von Worten durch den Diener des Gottes und Diener der Göttin (?) (22), deinen Sohn Hr-t3j.f-nht (II), seine Mutter ist $N s-N h m t-{ }^{-} w 3 j:$, Komm, mein vortrefflicher Vater, damit du dies siehst, was dein ältester Sohn Ns-Mnw (II) für dich getan hat, um zu veranlassen, daß du in der Nekropole zufrieden bist. Ich will in deinem Haus(halt) bleiben, indem ich wie die ,Finger' seines [des Hauses] (23) ältesten Sohnes bin. Er hat veranlaßt, daß ich der Bildhauer in deinem Grab bin (24).“

\section{B. Haupttext}

[Ein Königsopfer des ... Onuris-Sch]u, des Sohnes des Re, des Vaters der Götter, der Tefnut, der Tochter des Re, der Herrscherin in Heliopolis, der Mhjt, der Herrin von Wbn (5), der Großen zu Gast in Bhdt-[j3btt] (25) [...] 2 [... indem sie geben ... was der Ni]l aus seinem Quelloch [bringt] (26), wovon die Götter leben, angenehmen Wind (??) (27) [. . für den $\mathrm{Ka}$ des ...] 3 [. . hrp ले (28), Schreiber] der Zuteilung (?) der 4. Phyle (29) des Herrn des Hauses des Onuris, des 2. Propheten der Mhjt, der Herrin von $W b n$, des 4. Pro<pheten> [des OnurisSchu, des Sohnes des Re Dd-Jnj-hrt-jw.f- $n h$ (II), des Sohnes des gleichrangigen] 4 [Ns-Mnw (I), gerechtfertigt, des Sohnes des ...] (30), des Schreibers des Schatzhauses des Onuris $\underline{D} d-J n j-$ $h r t-j[w . f-n h(\mathrm{I})$, gemacht von der Hausherrin und Musikerin vom] 5 [Haus der Mhjt und des 
Onuris T3-srjt-Mhjt], der Tochter des [...] 6 Schreiber... [...]

\section{Anmerkungen}

1) Die Redeeinleitung $\underline{d} d-m d w$ jn kommt auf dieser Stele nicht weniger als dreizehnmal vor, mit sehr unterschiedlichen Schreibungen des jn: $7 \times<4,3 \times 4,1 \times 4(j<n>)$ und $2 \times$ sogar als $₫$ दै (offenbar ein Fehler für dsy .

2) Man beachte das Genetivverhältnis, nicht etwa „Osiris NN“. Die Statuette wird als Osiris(figur) des Toten bezeichnet. Allerdings hat M. Smith, Catalogue of Demotic Papyri in the British Museum, Vol. III, The Mortuary Texts of Papyrus BM 10507, 75-79, zu beweisen versucht, daß der Ausdruck Wsjr (n) NN grundsätzlich „Osiris des NN" bedeutet. Das scheint mir nicht überzeugend. Zunächst ist die Verbindung Wsjr $n N N$ fast ausschließlich auf demotische Texte beschränkt (wo man häufig ein „bedeutungsloses" $n$ findet), in hieroglyphischen und hieratischen Texten ist sie sehr selten, obwohl funeräre Texte, in denen diese Verbindung vorkommt, ganz überwiegend hieroglyphisch oder hieratisch geschrieben sind. Dennoch ist in einigen Fällen zweifellos von einer (bedeutungsgleichen) Variante $W$ sjr $N N / W$ sjr $n N N$ auszugehen, aber dort wird es sich um den Wechsel von indirektem Genetiv und Apposition handeln, wie er gerade vor Namen auch sonst belegt ist, etwa in Fällen wie $d m j(n)$ $N N$, der Ort NN“, vgl. Wb V, 455, 15-6; ZÄS 127 (2000), 28, wie das schon G. Vittmann, GM 83, 1984, 78; 80 (17), vorgeschlagen hatte. An der hier besprochenen Stelle dürfte dagegen der Genetiv in seiner eigentlichen, possessiven Bedeutung gemeint sein.

3) $\mathrm{Zu}$ diesem und den folgenden Titeln vgl. die Erläuterungen von $H$. de Meulenaere, CdE 29, 1954, 226-34.

4) Der Titel rmnj nsrt kommt außer auf den von de Meulenaere (op. cit., 228-9) zitierten Belegen auch noch auf der unpubli- zierten Schreiberstatue Kairo JE 37327 aus der 26. Dynastie vor.

5) Zur Ortsbezeichnung $W b n$ vgl. AEO, II, *37-8. Gegenüber der dort vorgeschlagenen Identifizierung mit Bhdt-j3btt hat de Meulenaere zur Vorsicht gemahnt (CdE 29,228, n. 3). Vielleicht ist $W b n$ ja ein Tempel oder Heiligtum in Bhdt-j3btt.

6) Vgl. CdE 29,223.

7) Das $f$ von $j w . f-$ - $n h$ könnte nachträglich hinzugefügt worden sein.

8) Die Pluralstriche gehören eigentlich vor das Suffix. Zur Bedeutung von $\underline{d} \underline{d} \underline{d} t . f \mathrm{~s}$. u.

9) jtj.t.t(n), vgl. M. Lichtheim, Maat in Egyptian Autobiographies and Related Studies, OBO 120, 1992, 176-7. „Vater" hier auch in allgemeiner Bedeutung als ,Vorfahr“"

10) Wörtlich „unter ihm“.

11) Gemeint ist Bhdt-j3btt, vermutlich das heutige $\mathrm{Nag}^{\mathrm{c}}$ el-Mescheich, vgl. AEO; II, *36-7; L $\ddot{A}$ IV, 107.

12) Das Suffix . $s(n)$ kann sich nur auf die beiden Ortsnamen $\underline{T} n j$ und $B h d t$ beziehen; gemeint ist natürlich , in ihren Tempeln".

13) Dieses zweite Suffix.$s(n)$ wird sich entweder wiederum auf die beiden Ortsnamen beziehen oder auf ntrw. Sachlich besteht kaum ein Unterschied, denn die Handlungen eines Priesters für einen Ort würden ja den Tempeln bzw. Göttern darin gelten.

14) Trotz der Schreibung wohl nur als nwtjw.f zu verstehen, kaum als nwtjw nwt.f ,Städter seiner Stadt"،

15) Der folgende Abschnitt ist auch bei de Meulenaere, CdE 29,235 übersetzt, der auch schon die offensichtliche Emendation von $k 3 r$ zu $k 3 t$ (vermutlich durch Assoziation mit $k 3 r$ „Kapelle“ bedingt) vorgeschlagen hatte.

16) $\mathrm{Zu} m n j$ im Sinne von „begraben“ s. Hornung, Sonnenlitanei, II, 98, n. 11.

17) Vgl. Wb II,387,25-6. Vermutlich ist die Gesamtdauer der Begräbnisfeierlichkeiten gemeint, kaum nur die „Mundöffnung “, bei der zumindest für die Spätzeit eine Dauer von vier Tagen überliefert ist (vgl. Sethe, Zahlworte, 32).

18) Die beiden Personen sind wohl nur ein ungewöhnliches Determinativ des Plurals von 
$m j$, kaum ein eigenes Wort rmtw („kommt, Leute ${ }^{6}$ ).

19) Haplographie $r<n>. n$; ein Plural $r n w$ (ohne Suffix) wäre wenig sinnvoll.

20) Das ॰ unter ‥m wird zu $\underline{d} t$ gehören, d. h. es

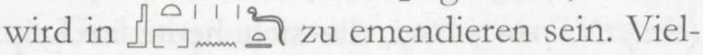
leicht hatte der Schreiber an st tn gedacht.

21) Die Ergänzung $<z 3>$ hinter D $d$-Jnj-ḥrt-jw.f' $n h$ ergibt sich aus dem Vergleich mit einer Beischrift auf der Stele in Leiden, auf der gleichfalls der Urenkel des Statuenbesitzers dargestellt ist (rechts unten), s. de Meulenaere, CdE 29,224-6. Und auf der Leidener Stele wird gleichfalls der Name seiner Mutter hinter der Angabe mwt.f weggelassen, obwohl dort genug Platz gewesen wäre (die untere Hälfte der Kolumne ist nicht beschrieben). Es scheint also irgendeinen Grund dafür zu geben, daß der Name der Mutter nicht erscheint.

22) Zum Titel 97 Q 29,227, n. 1, der darin (unter Vorbehalt) eine Kurzform für hm sj3 hm $3 \mathrm{~h}$ vermutet hat. Die beiden anderen Belege, die er zitiert, haben die Form 17 Q. Diese Variation erinnert zunächst an den zusammengesetzten Titel $h m h \underline{d} t h \quad h m H r(w r w 3 \underline{d} t j)$, der zum einen mit Ausschreibung beider nomina regentis $(\mathrm{hm})$ belegt ist (vgl. z. B. de Meulenaere, $\operatorname{CdE} 68,1993,60)$, häufiger aber in einer kontrahierten Form $\mathrm{hm} h \underline{h d t}(\mathrm{hm}) \mathrm{Hr}$ ( $w r$ w $\underline{d} t j)$, vgl. Cl. Traunecker, in: Gs Quaegebeur, 1215ff. Auch hier könnte es sich um Kurz- und Langform handeln, und da ใh sehr häufig die Lesung ntrt hat, ist der Titel vielleicht am ehesten als $h m$ ntr $(\mathrm{hm})$ ntrt zu verstehen. Falls es sich um einen abydenischen bzw. thinitischen Titel handelt, könnten ntr und ntrt auf Onuris(-Schu) und Mehit verweisen.

23) Das Suffix ist offenbar auf $p r$ bezogen; die Formulierung soll wohl besagen, daß Ns-Mnw II jetzt das Familienoberhaupt ist.

24) Und insofern der „Finger" (eine ähnliche Metapher wie „der verlängerte Arm“?) seines ältesten (Halb)Bruders, in dessen Auftrag er das Grab dekoriert.

25) Etwas anderes als Bhdt-j3btt kommt hier eigentlich nicht in Frage, aber die Spuren am
Ende der Zeile passen nicht unbedingt zu dieser Lesung.

26) In der Lücke muß auf jeden Fall dj.sn gestanden haben, und von den Gaben ist noch [jn(nt) $H^{\top} p$ ]j $m$ tpht.f zu rekonstruieren.

27) Eine entstellte Form (bzw. Gruppierung) von $t 3 w n d m[n m h j t \ldots]$ ?

28) Siehe de Meulenaere, CdE 29,230-1. Ergänzungen nach der Stele in Leiden (ibid., 222).

29) Zum Titel $z \check{s} d \check{s} / t 3 \check{s}$ s. ibid., 229-30.

30) In Analogie zur Leidener Stele sollte man am Anfang der Titulatur des Großvaters $z \check{S}$ $j m j-r^{\prime} k 3$ erwarten (vgl. de Meulenaere, CdE 29,231, n. 2), aber der Rest zu Beginn von Z. 4 ist עy zu lesen und kann nicht zu jmj-r' $k 3$ gehören.

Obwohl alle 13 Beischriften von $\underline{d} d-m d w$ eingeleitet werden, folgt nur in zwei Fällen tatsächlich eine Rede der betreffenden Person, und zwar im Falle des ältesten Sohnes $N s-M n w$ II und in dem des Bildhauers $H r-t 3 j . f-n h t$ II. Beide Reden haben zum einen „familiäre“ Angelegenheiten als Thema, zum anderen beziehen sie sich unmittelbar auf die bildlich dargestellte Prozession. Zunächst zu den familiären Dingen:

$H r$-t3j.f-nht (II) sagt, daß ihn das nunmehrige Familienoberhaupt Ns-Mnw II als Bildhauer im $h w t$ des Vaters beschäftigt hat (als sein „Finger“, der nach seinen Vorgaben arbeitet), und dieses $h w t$ kann nach Lage der Dinge nur das Grab sein. Er wird also für die Dekoration des Grabes verantwortlich gewesen sein (sofern es dekoriert war), und vermutlich hat er auch die Stelen hergestellt. Er betont, daß er im Haus(halt) seines Vaters (der jetzt eben seinem ältesten Bruder untersteht) bleiben darf. Das scheint für ihn als (Halb)Bruder also nicht selbstverständlich gewesen zu sein.

$N s-M n w$ spricht zum einen seine $\underline{d} \underline{d} \underline{d} 3 t r$ - $d r . s$ an, und dann auch noch im besonderen seine Kinder $(\underline{h} r d w . f)$. Mit $\underline{d} 3 \underline{d} 3 t . f r \underline{d} r . s$ werden zunächst alle diejenigen gemeint sein, die hier am Totenkult für seinen Vater beteiligt sind, also die Großfamilie des Sprechers. Es wäre allerdings nicht auszuschließen, daß auch noch andere Priester daran teilnahmen, die zwar als nicht zur Familie gehörig nicht abgebildet wurden, aber 
gleichfalls angesprochen sind. Neben der Großfamilie (bzw. allen Kultbeteiligten) werden die Kinder des Ns-Mnw (II) im besonderen angesprochen, obwohl sie ja schon zu dieser ersten Gruppe gehören. Die sich anschließende Rede ist eine Aufforderung zur Beteiligung am Totenkult für seinen Vater, da dieser selbst auch ein frommer Mann gewesen ist. Man wird annehmen dürfen, daß die besondere Anrede an seine Kinder eine indirekte Aufforderung ist, später auch für den Sprecher so zu handeln, und das wird etwas später ja auch explizit gesagt. Nach der kurzen Darstellung der Priestertätigkeit des Verstorbenen, die sich über 60 Jahre erstreckte, werden die Angesprochenen aufgefordert, ebenso zu handeln, damit auch sie später einen Totenkult haben. Im Hinblick auf die Familie sagt $N s-M n w$ seinen Leuten also, ein wie vorbildlicher Priester sein Vater war, daß er deshalb ein fortdauerndes Gedenken verdient hat und daß die Familie sich daran ein Beispiel nehmen soll, damit es ihnen später ebenso ergehe.

Auf die Darstellung bezogen sind die einleitenden Passagen der beiden Reden. Hr-t3j.f-nht beginnt folgendermaßen: „Komm, mein vortrefflicher Vater, damit du dies siehst, was dein ältester Sohn Ns-Mnw für dich getan hat, damit du in der Nekropole zufrieden bist." Das, was der Vater sich ansehen soll, kann doch nur das Grab bzw. die Grabausstattung sein (u. a. auch die Dinge, die in der Darstellung gezeigt werden). Demnach sollte es sich bei dieser Darstellung um das Grabgeleit des Vaters handeln. Tatsächlich sind derartige „Prozessionen“, in denen u. a. Götterstandarten getragen werden, in der Spätzeit als Darstellung des Trauergeleits nicht unüblich ${ }^{17}$, vgl. etwa die entsprechenden Szenen in Gräbern ${ }^{18}$, auf Särgen ${ }^{19}$ und Papyri ${ }^{20}$. Diese Götterstandarten sind von den königli-

${ }^{17}$ S. P. Barthelmeß, Der Übergang ins Jenseits in den thebanischen Beamtengräbern der Ramessidenzeit, SAGA 2, 1992, 165: „Die Männer des Trauergeleits tragen nun [nach der 21. Dynastie] häufig Standarten".

G. Lefebvre, Le tombeau de Petosiris, Kairo 1923, III, pl. XXVIII-XXXIV; I, 128-30.

${ }^{19}$ J. Capart, CdE 18, 1943, Fig. 28; 32 (zwei Särge aus Hildesheim).

${ }^{20}$ R. Lepsius, Das Todtenbuch der Ägypter, Leipzig 1842, Taf. I-III. chen Bestattungen in die ,privaten" übernommen worden ${ }^{21}$. Nach den Bestattungszeremonien wurden sie offenbar im Grab deponiert ${ }^{22}$. Gut erhalten haben sich die Standarten aus dem Grab des jtj-ntr hm(-nțr) Jmn-Re njswt ntrrw (etc.) Ns-p3wtj-t3wj, die sich heute in Leipzig und Turin ${ }^{24}$ befinden und aus der späteren Ptolemäerzeit stammen ${ }^{25}$. Bei anderen Exemplaren aus Bronze ${ }^{26}$ und Silber ${ }^{27}$ ist es nicht sicher, ob sie aus dem funerären Bereich stammen.

Auch die Figur, die durch die Beischrift als Osiris des Toten identifiziert wird, muß etwas mit seiner Bestattung zu tun haben und dürfte zur Grabausstattung gehört haben. Handelte es sich dagegen um eine Statue des Toten, die im Tempel von Thinis aufgestellt werden sollte ${ }^{28}$, wäre ihre Form unerklärlich; Tempelstatuen von Privatleuten sehen anders aus. Und auch mit dem Gott Onuris kann sie nichts zu tun haben ${ }^{29}$; warum sollte eine Statue des Toten die Krone des Onuris tragen oder gar mit Onuris identifiziert werden? Ebensowenig wird man aus der

${ }^{21}$ Vgl. LÄ II,714; Bonnet, RÄRG, 254; Erman, Die Religion der Ägypter, Berlin/Leipzig ${ }^{4} 1934,453$ (Anm. 2 zu p. 290). Sie werden aber auch im Götterkult verwendet, vgl. etwa Mariette, Dend., I, 9; IV,33.

${ }^{22}$ Vgl. auch Erman, op. cit., 290: „In anderen Gräbern trifft man hölzerne Standarten mit den Figuren der Göttertiere an, wie man sie in den Prozessionen voranträgt, um den Weg zu bereiten."

Ägyptisches Museum der Universität Leipzig, Inv.-Nr. 2903 und 2905, s. G. Steindorff, Zwei Holzbilder des Wolfsgottes Up-uaut, in: Mélanges Maspero, MIFAO 69/II, Le Caire 1938, 841-846; H. Felber, in: R. Krauspe, Katalog Ägyptischer Sammlungen in Leipzig, Bd. 1: Statuen und Statuetten, Mainz 1997, 98-100.

${ }^{24}$ Nr. 914, 986, 1009, 6963, 6963 bis, 6363ter, s. G. Botti, Statuette per stendardi funerari del Museo Egizio di Torino, in: Studi in onore di Ugo Enrico Paoli, Florenz 1955, 145-8; nachgedruckt in Omaggio a Giuseppe Botti, Mailand 1984, 65-72.

${ }^{5}$ Das Grab des Vaters Wsjr-wr wird in einem griechischen Papyrus aus dem Jahr 113 v. Chr. erwähnt, vgl. J. Quaegebeur, Prêtres de cultes thébains à la lumière de documents égyptiens et grecs, BSFE 70/71, 1974, 43-4; ders., in: Aspekte spätägyptischer Kultur, FS Winter, AegTrev 7, Mainz 1994, 215-217, vgl. auch Felber, in: Krauspe, op. cit., 100.

${ }^{26}$ Petrie, Nebesheh, p. 26; pl. VII, 3.

${ }^{27}$ H. Schäfer, Ägyptische Goldschmiedearbeiten, Berlin 1910, p. 69-70, Abb. 75, Taf. 16 (Nr. 103).

${ }^{28}$ So de Meulenaere, CdE 29,223.

${ }^{29}$ Gegen de Meulenaere, loc. cit. 
Art dieser Figur schließen dürfen, „daß man dem Toten nachgerade göttliche Ehren erwies $^{66^{30}}$. Es wird sich vielmehr um eine „PtahSokar-Osiris-Statuette" handeln, wie schon M. Raven vermutet hatte ${ }^{31}$, also um eine Figur, die im Grab deponiert war, nicht im Tempel ${ }^{32}$. $\mathrm{Da}$ an diesen Statuetten im Zusammenhang mit der Bestattung gewisse Zeremonien vorgenommen wurden, geht aus ihrer Darstellung auf Särgen hervor. Auf dem Sarkophag Kairo CG 41026 eines Harsiese steht Anubis hinter einer solchen Figur (die als Osiris [des?] Harsiese bezeichnet wird), während ihr der Jwn-mwt.f gegenübersteht und ihre Stirn salbt ${ }^{33}$. In der Inschrift darüber wird der Tote als $j m 3 h w$ bei Osiris und Ptah-Sokar-Osiris bezeichnet, und auch eine der flankierenden Opferformeln ist im Namen des Ptah-Sokar-Osiris (und des Anubis). Auf zwei Särgen in Hildesheim werden im Beisein eines Vorlesepriesters bestimmte Riten an solch einer Ptah-Sokar-Osiris-Statue vollzogen ${ }^{34}$. J. Capart hat das so gedeutet, daß „les rites préliminaires à l'ensevelissement fixaient l'âme du mort à une statue osirienne " " Auf jeden Fall aber zeigen diese beiden Särge einen engen $\mathrm{Zu}$ sammenhang zwischen diesen Zeremonien und dem Totengeleit, bei dem Götterstandarten getragen wurden, denn dieses Standartengeleit wird auf ihnen ebenfalls abgebildet ${ }^{36}$. Es scheint sich also um rein funeräre Zeremonien zu handeln, und man sollte annehmen, daß sie sich dann im Grab bzw. bei der Balsamierungsstätte abgespielt haben. Dazu scheint aber die Rede des Ns-Mnw im Widerspruch zu stehen, denn er sagt mit eindeutigem Bezug auf die dargestellte Prozession: „Gebt eure Arme unter euren Vater in T3-wr. Eure Arme sollen nicht erlahmen, wenn ihr ihn tragt, um ihn nach Thinis und $B h d t$ gelangen zu lassen im Verlauf jeden Tages. Vollzieht für ihn seine Riten vor den Göttern in ihnen [ = Thinis und Bhdt] ]. Diese Sätze hatten de Meulenaere verständlicherweise dazu geführt, die Figur für eine Tempelstatue zu halten, aber ihre Form sowie der ganze Zusammenhang sprechen dagegen, Statue und Prozession sind deutlich funerär. Dennoch geht die Prozession zunächst offenbar zum Tempel, und dort werden bestimmte Zeremonien für den Toten vollzogen, wie es $N s-M n w$ unmißverständlich sagt. Man wird sich das nur so erklären können, daß die Figur im Tempel ,geweiht" worden ist, bevor sie dann im Grab deponiert wurde. Und diese Weihung im Tempel dürfte es dann dem Toten erlaubt haben, „täglich“" zum Tempel zu kommen, vermutlich um an Festen u. ä. teilzunehmen, denn das $m$ hrt hrw nt $r^{r} n b$ kann sich ja unmöglich auf die dargestellte Prozession selbst beziehen. Es wird bei dieser Darstellung also darum gehen, daß die Gegenstände der Grabausstattung vor dem eigentlichen Begräbnis im Tempel geweiht wurden.

\section{SUMMARY}

Republication of a stela fragment in the Cairo Museum, originally from Abydos and dating to the 4th-3th century B.C. Above the main text is a representation of the male offspring of the deceased in a kind of procession, bearing objects belonging to the funerary cult. Nevertheless, the related texts speak of a procession to the temple of Thinis. These objects were possibly consecrated in the temple before they were brought into the tomb.

${ }^{30}$ So P. Munro, Totenstelen, 111, der das daraus schließt, daß die Figur die „Gestalt des Osiris mit der Federkrone" hat.

${ }^{31}$ OMRO 60, 1979, 284 (mit n. 237).

${ }^{32}$ S. M. J. Raven, Papyrus-Sheaths and PtahSokar-Osiris-Statues, OMRO 60, 1979, 251-96.

${ }^{33}$ A. Moret, Sarcophages de l'époque bubastide à l'époque saïte, CG, Kairo 1913, 258-9; pl. XXVIII.

J. Capart, „A propos du cercueil d'argent du roi Chechonq", CdE 18, 1943, 191-8.

${ }^{35}$ Ibid., 194.

${ }^{36}$ CdE 18, Fig. 28; 32. 


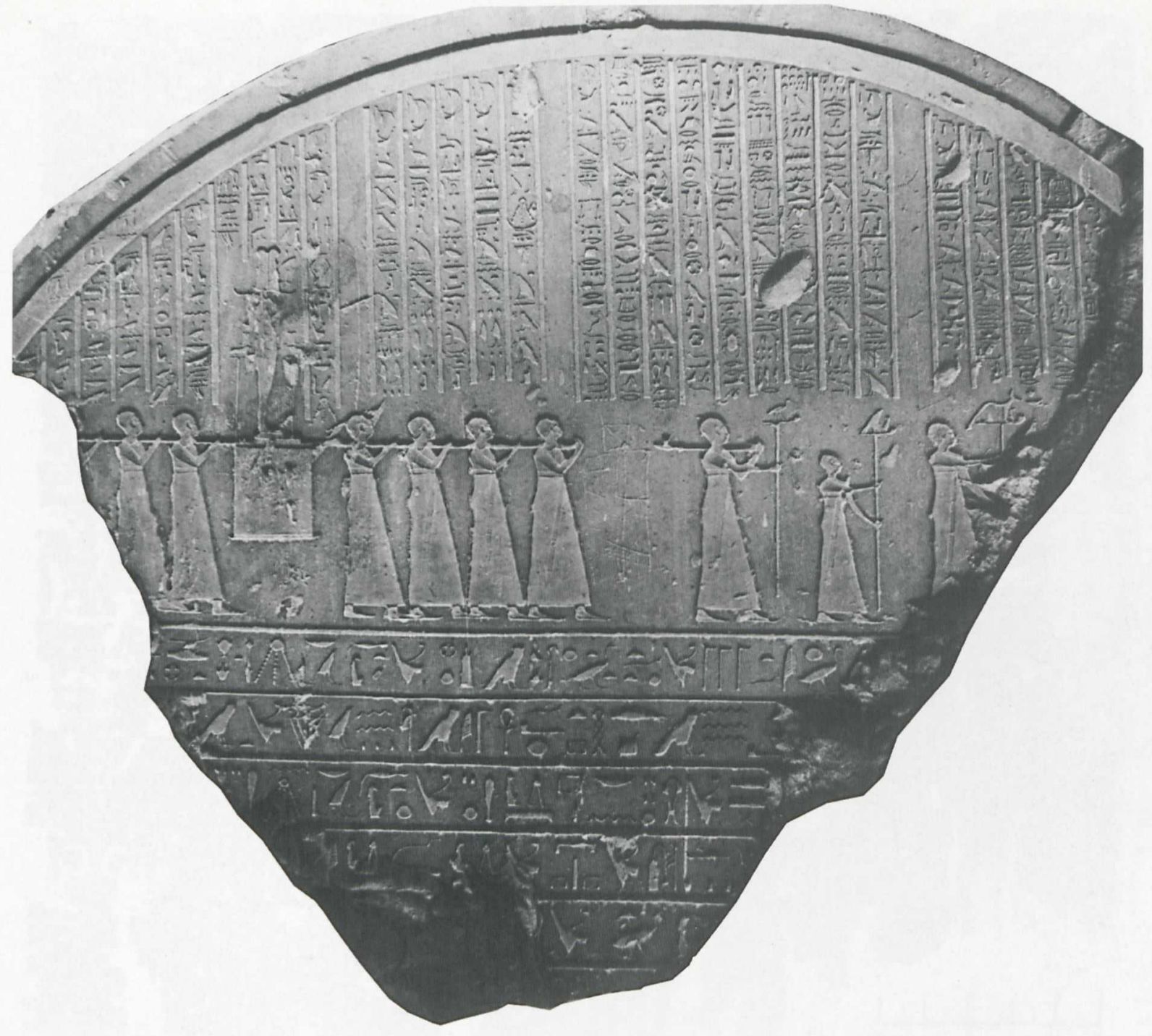

Stelenfragment Kairo TN 30/5/24/1 (zu Jansen-Winkeln, Familie im Totenkult) 